\title{
Sequence analysis of cultivated strawberry (Fragaria $\times$ ananassa Duch.) using microdissected single somatic chromosomes
}

Tomohiro Yanagi $^{1 *}$, Kenta Shirasawa $^{2}$, Mayuko Terachi $^{1}$ and Sachiko Isobe ${ }^{2}$

\begin{abstract}
Background: Cultivated strawberry (Fragaria $\times$ ananassa Duch.) has homoeologous chromosomes because of allooctoploidy. For example, two homoeologous chromosomes that belong to different sub-genome of allopolyploids have similar base sequences. Thus, when conducting de novo assembly of DNA sequences, it is difficult to determine whether these sequences are derived from the same chromosome. To avoid the difficulties associated with homoeologous chromosomes and demonstrate the possibility of sequencing allopolyploids using single chromosomes, we conducted sequence analysis using microdissected single somatic chromosomes of cultivated strawberry.

Results: Three hundred and ten somatic chromosomes of the Japanese octoploid strawberry 'Reiko' were individually selected under a light microscope using a microdissection system. DNA from 288 of the dissected chromosomes was successfully amplified using a DNA amplification kit. Using next-generation sequencing, we decoded the base sequences of the amplified DNA segments, and on the basis of mapping, we identified DNA sequences from 144 samples that were best matched to the reference genomes of the octoploid strawberry, F. $\times$ ananassa, and the diploid strawberry, F. vesca. The 144 samples were classified into seven pseudo-molecules of $F$. vesca. The coverage rates of the DNA sequences from the single chromosome onto all pseudo-molecular sequences varied from 3 to $29.9 \%$.
\end{abstract}

Conclusion: We demonstrated an efficient method for sequence analysis of allopolyploid plants using microdissected single chromosomes. On the basis of our results, we believe that whole-genome analysis of allopolyploid plants can be enhanced using methodology that employs microdissected single chromosomes.

Keywords: Strawberry (Fragaria $\times$ ananassa Duch.), Allopolyploid, Chromosome microdissection, Sequence analysis, Mapping

\section{Background}

Cultivated strawberry (Fragaria $\times$ ananassa Duch.) is one of the most popular fruit crops worldwide and is grown across a wide range of regions from subarctic to tropical [1]. Cytogenetic studies have determined that the chromosome number of somatic cells of cultivated strawberry is $56[2-5]$. In addition, cultivated strawberry is an allo-octoploid, having three complex genome compositions: $\mathrm{AABBBBCC}$ [6], $\mathrm{AAA}^{\prime} \mathrm{A}^{\prime} \mathrm{BBBB}$ [7], or $\mathrm{AAA}^{\prime} \mathrm{A}^{\prime} \mathrm{BBB}^{\prime} \mathrm{B}^{\prime}$ [8]. In addition, Tennessen et al. [9] and

\footnotetext{
*Correspondence: yanagi@ag.kagawa-u.ac.jp

${ }^{1}$ Faculty of Agriculture, Kagawa University, Miki-cho, Kita-gun, Kagawa

761-0795, Japan

Full list of author information is available at the end of the article
}

Sargent et al. [10] have recently proposed updated models-AvAvB1B1B2B2BiBi and AA, bb, X-X, X-X, respectively. The amount of DNA within a haploid nucleus of cultivated strawberry has been estimated at $708-720 \mathrm{Mb}$ [11]. On the basis of this data, the average DNA size of a single chromosome can be calculated as approximately 25-27.8 $\mathrm{Mb}$. In addition, the mean chromosome length of some wild octoploid strawberries has been determined to be approximately $1 \mu \mathrm{m}$ [12]. The size of a single chromosome in cultivated strawberry appears to be very small as likely as that in rice and Arabidopsis thaliana.

Allopolyploidy is a problem when conducting genetic analyses, because the presence of similar sub-genomes has led to multiple alleles and complex segregation 
ratios [13]. Thus, theoretical genetic analysis and breeding in cultivated strawberry are extremely difficult based Mendel's law of inheritance. To resolve this problem, the determination of accurate base sequences covering the entire genome of cultivated strawberry is needed to construct a high-density linkage map. If this can be achieved, many DNA markers that follow Mendel's law of inheritance could be discovered, and theoretical breeding using genomic selection could be performed with high reliability. Hirakawa et al. [14] reported the draft genome sequences of a Japanese cultivated strawberry using next-generation sequencing (NGS). The base sequences of entire DNA segments from the strawberry 'Reiko' were determined, and approximately $70 \%$ of these sequences were assembled into larger DNA scaffolds. However, to date, the sequences have not been assigned to chromosomes. Moreover, $30 \%$ of the genome remains unsequenced, because of the existence of sub-genomes that have homoeologous chromosomes. For example, two homoeologous chromosomes that belong to different sub-genomes may have similar but slightly different base sequences. Thus, when performing standard genome assembly, it is difficult to accurately assign such sequences to an appropriate pseudo-molecular chromosome. To overcome this problem, it is necessary to develop an alternative method of sequence analysis for allopolyploids.

In the present study, in order to avoid difficulties arising from the occurrence of homoeologous chromosomes, we attempted to conduct sequence analysis using microdissected single somatic chromosomes. Although a technique for chromosome microdissection was developed in the 1980s [15], it has been unpopular for elucidating the base sequences of whole genomes. Scalenghe et al. [16] initially microdissected a small segment of the chromosome in Drosophila melanogaster, and demonstrated the possibility of directly generating DNA segments. As discussed by Zhou and $\mathrm{Hu}$ [17], many studies have been conducted using chromosome microdissection for human and animal cells, but a smaller number have been performed in plants because chromosome sample preparation is more difficult in plants. In higher plants, Sandery et al. [18] first reported microdissection and DNA generation of B-chromosomes in rye. Subsequently, chromosome microdissection has been used in several facets of genomic research, including (1) genetic linkage map and physical map construction, (2) generation of probes for chromosome painting, and (3) generation of chromosome-specific expressed sequence tag libraries [19]. However, to date, few studies have used chromosome microdissection to determine base sequences of whole genomes in plants.
The purpose of the present study was to determine the effectiveness of sequence analysis using single chromosomes for a typical allopolyploid cultivated strawberry plant. Furthermore, we also examined the possibility of amplifying DNA from a very small single chromosome using a DNA amplification kit.

\section{Results and discussion \\ Efficiency of the microdissection system}

By way of illustrating chromosome microdissection in the cultivated strawberry, the equipment used and pictures of the chromosome and somatic cells are shown in Fig. 1. Fifty-four chromosomes were observed in somatic cell (Fig. 1b) under a light microscope, and used for microdissection. The remaining two chromosomes were missing. In Fig. 1c, a single microdissected chromosome can be seen at the terminal portion of a glass needle. The single chromosome surrounded by a red circle (Fig. 1b) subsequently disappeared (Fig. 1d), and the remaining 53 were individually selected (Fig. 1e). All chromosomes were eventually selected and removed from the somatic cell (Fig. 1f). Although the time from installing a new glass needle to placing a single chromosome in a PCR tube was not measured, on the basis of the total time of the microdissection and the number of chromosomes microdissected, we estimate that it probably took approximately 2 min to dissect a single chromosome. In general, chromosome microdissection can be performed using (1) a light microscope with a micromanipulator system and glass needle, and (2) a light microscope with a laser capture microdissection system. In the present study, a light microscope was used in conjunction with a micromanipulator system and glass needle. Using this system, because chromosome could be moved when it was pushed carefully by the apex of the glass needle, partially overlapped chromosomes could easily be separated and individually selected, which would not have been possible using a laser capture microdissection system. Further, a flow-cytometry and sorting devices can correct and take some same-sized chromosomes. However, using such a device might result in contamination from similar-sized homoeologous chromosomes because of its limited sensitivity, as indicated by Zhou and $\mathrm{Hu}$ [17]. Recently, Capal et al. [20] reported that it was possible to take one $3 \mathrm{~B}$ chromosome of wheat by the flow cytometry and sorting devices. However, the paper did not show the method to confirm whether one chromosome was precisely selected in one tube or not. In addition, we thought that the chromosomes of the cultivated strawberry were very small and similar size unlike the chromosomes of wheat. For this reason, the flow-cytometry and sorting devices were not used in the present 

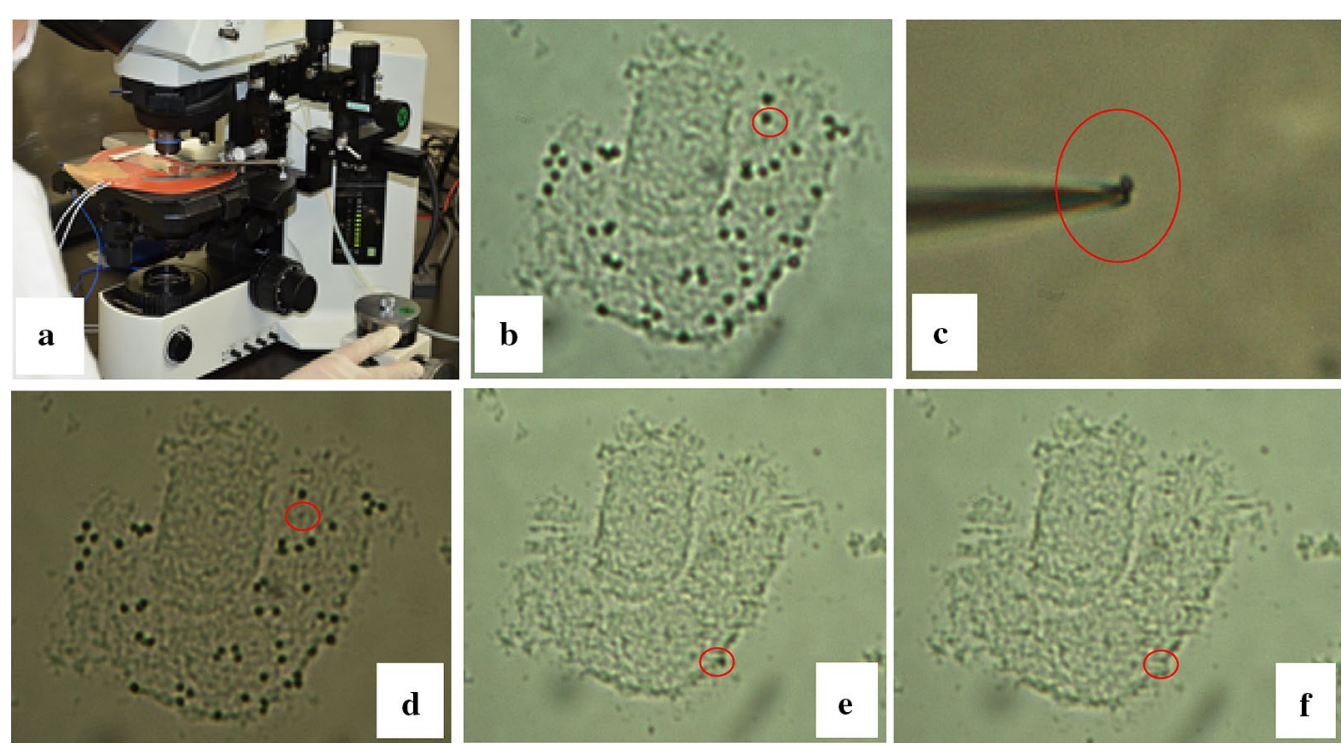

Fig. 1 The microdissection equipment used in the present experiment and some pictures of chromosome and somatic cells of the cultivated strawberry. a Microdissection equipment which was used in the present experiment, $\mathbf{b}$ chromosome image of the cultivated strawberry; the red circle shows the chromosome which will be selected, $\mathbf{c}$ a single dissected chromosome on the glass needle, $\mathbf{d}$ the chromosome in $\mathbf{b}$ is disappeared, e the red circle shows the final chromosome of the somatic cell, and $\mathbf{f}$ all chromosomes were selected in the somatic cell

research. Chromosome microdissection with the micromanipulator system used in the present study generally requires an experienced operator; however, under the guidance of a person skilled in chromosome microdissection, a beginner can select a single chromosome after practicing just two or three times. Furthermore, since the size of cultivated strawberry chromosomes (approx. full length $=1 \mu \mathrm{m}$ ) appears to be at the lower limit for microdissection, chromosomes larger than those of cultivated strawberry can be selected using the same method.

\section{Confirmation of DNA amplification from a single chromosome of cultivated strawberry}

In the present study, 310 chromosomes were removed from 10 metaphase somatic cells of cultivated strawberry. To confirm the efficiency of the DNA amplification kit for amplifying single chromosome DNA of cultivated strawberry, the DNA concentration of each amplified sample was measured. The DNA concentration of the samples differed from almost zero to more than $500 \mathrm{ng} / \mu \mathrm{L}$ (Fig. 2). Approximately $60 \%$ of the samples were amplified to more than $50 \mathrm{ng} / \mu \mathrm{L}$.

To clarify the components of each amplified DNA segment, the base sequences of the 288 samples with higher concentrations of DNA were decoded by NGS. For each sample, 1000 reads were extracted from the sequence data and mapped onto strawberry (FAN_r1.1 F. vesca), human, bacterial, and nematode reference genomes, among others. The mapping results for the 288 samples are shown in Fig. 3. Of these, the amplified DNA segments from 144 samples had more than $50 \%$ of reads that matched the reference genome of strawberry. The maximum matched value of 983 reads was recorded for sample FaMD-4-A10. These results clearly demonstrated that the Illustra Single Cell GenomiPhi DNA Amplification kit could amplify the DNA sequence of the strawberry chromosome by following the manufacturer's protocol, although DNA in approximately half

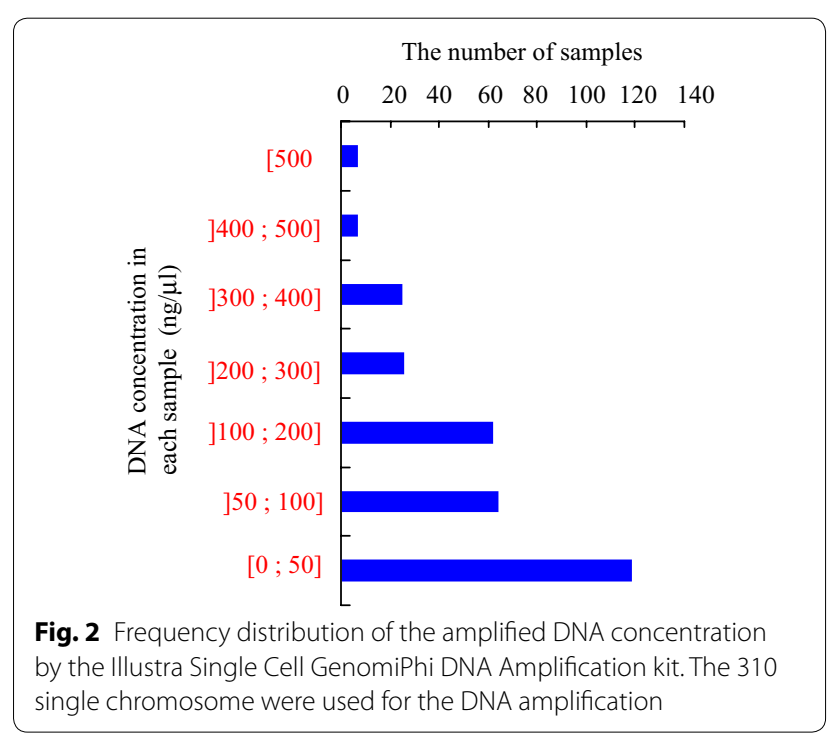




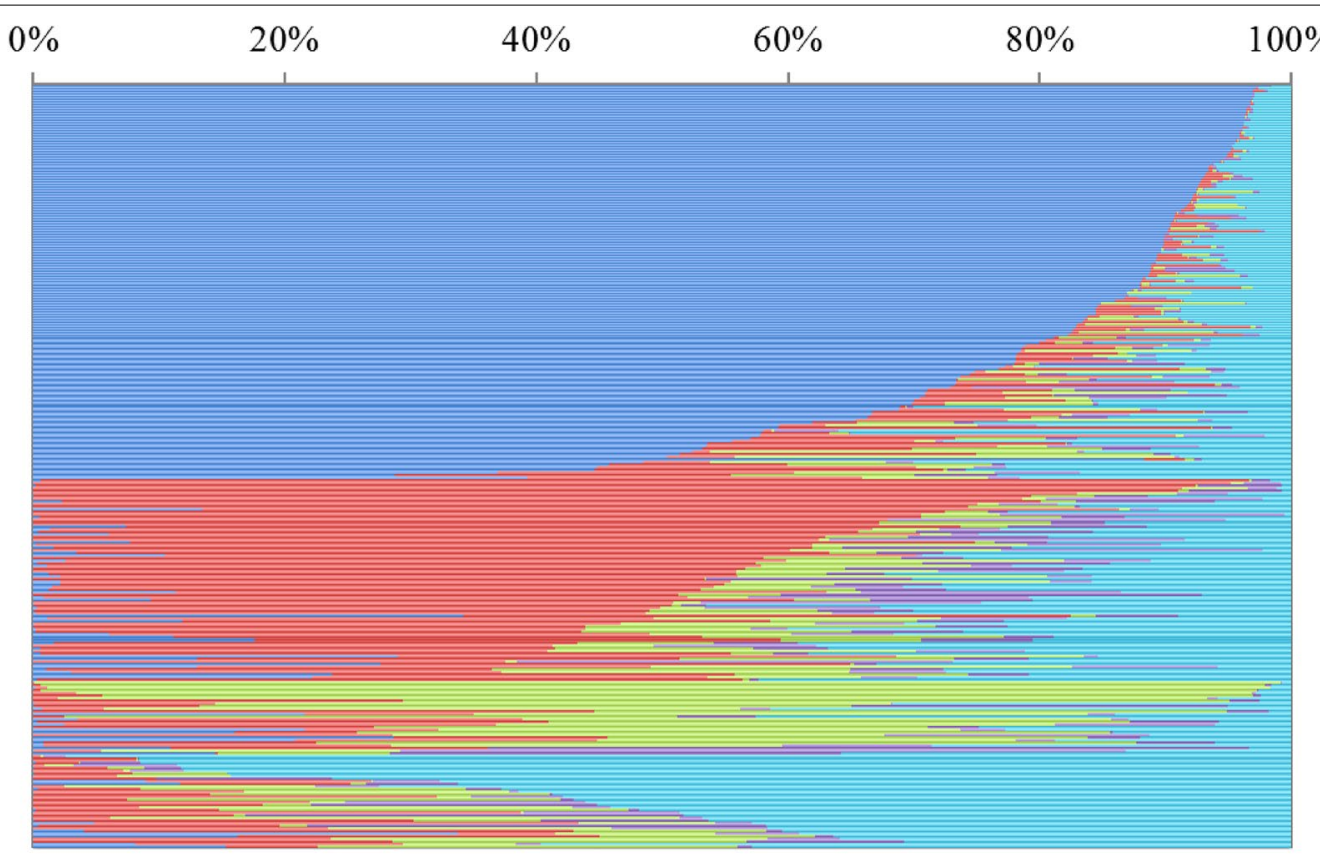

- Strawberry $\square$ Human $\square$ Bacteria and nematode $₫$ Other organisms $₫$ No Hits

Fig. 3 The results of mapping onto the reference genome of strawberry (FAN_r1.1 + F. vesca), human, bacteria, nematode and other organisms. The 1000 reads were extracted from the sequence data and mapped on the reference genome data. The 288 mapping data were summarized in this figure. Each thin bar exhibited the data of each sample

the samples was not amplified (Fig. 3). Conventionally, a linker adaptor-mediated PCR (LA-PCR) method $[15,21-23]$ and a degenerate oligonucleotide-primed PCR (DOP-PCR) method [24, 25] have been used for amplification of DNA segments obtained by chromosome microdissection. In addition, Seifertova et al. [26] reported a different method of chromosome DNA amplification using a different DNA amplification kit. In comparing these methods, the method presented here using the kit has some notable advantages, namely, the ease of sample preparation, shorter amplification time, and lower rate of unexpected amplification of contaminated DNA. On the basis of these features, we believe that the kit is appropriate for amplifying DNA from single microdissected chromosomes of cultivated strawberry. However, in the remaining 144 samples, the amplified DNA segments matched more with the reference genomes of humans, bacteria, nematodes, and other organisms than those of strawberry. The reason for this failure is probably that the microdissected chromosome was not appropriately placed into the $1 \mu \mathrm{L}$ phosphate-buffered saline (PBS) contained within the PCR tube. In this respect, it is generally very difficult to place a chromosome in the small amount of liquid, and accordingly, there is a necessity to refine this procedure in order to increase the number of samples with amplified DNA segments from microdissected chromosomes. Interestingly, chloroplast and mitochondrial DNAs were rarely amplified using the present method (i.e., organelle DNA sequences constituted approximately $0.21 \%$ of the amplified DNA).

\section{Mapping onto pseudo-molecules of the diploid Fragaria vesca, one of the genome donors in cultivated strawberry}

To examine the similarity of DNA sequences between microdissected single chromosomes and seven different pseudo-molecules (Fvb1 to Fvb7) of $F$. vesca, mapping was conducted using the reference genome of $F$. vesca, because no pseudo-molecules of the cultivated strawberry have been developed. The 144 sets of sample data were sorted according to the order of the best-matched pseudo-molecule from Fvb1 to Fvb7 (Fig. 4). Among these samples, 19, 18, 18, 21, 23, 17, and 28 samples best matched with Fvb1, Fvb2, Fvb3, Fvb4, Fvb5, Fvb6, and Fvb7, respectively. On the basis of these results, the dissected single chromosomes were identified as homoeologous to the pseudo-molecules in F. vesca. Furthermore, each sample that was best matched to any pseudo-molecule had $40-80 \%$ unmapped reads. However, as shown in Fig. 3, approximately $50-98 \%$ of the reads matched with the references genome of strawberry (FAN_r1 $1+F$. $v e s c a$ ). On the basis of these two values, the unmapped reads in Fig. 4 appeared to include some of the reads that 


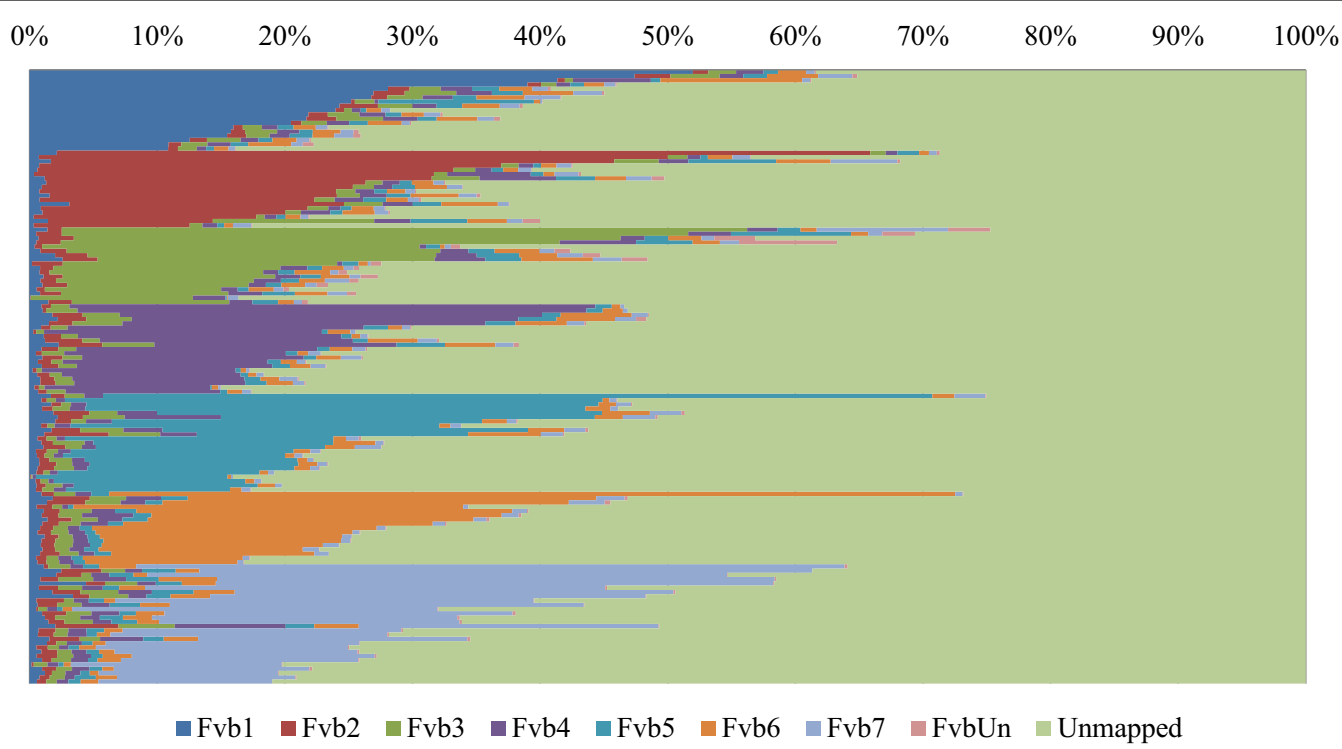

Fig. 4 The results of mapping onto pseudo-molecules of F. vesca $(2 n=14)$, that is one of the genome donors in the cultivated strawberry. The DNA segments that were obtained from a micro-dissected single chromosome, decoded the sequences by the NGS. Every DNA sequence was classified into Fvb1 to Fvb7 of pseudo-molecules in F. vesca, FvUn of unassigned sequence in F. vesca and unmapped one. The percentage of the reads in every pseudo-molecular were calculated, and sorted according to the higher order of the Fvb1 to Fvb7. Then the data were summarized in this figure. Each thin bar exhibited the data of each sample

originated from the dissected single chromosomes of cultivated strawberry. Thus, it is possible that the assignment of some unmapped reads could be important for clarifying the entire sequence of a single chromosome of cultivated strawberry.

To clarify the source of the unmapped reads, therefore, we investigated the origins of the chromosome samples, since the genome of $F . \times$ ananassa is supposed to contain those of the probable progenitors, e.g., $F$. vesca and $F$. iinumae. A total of 21 samples, three best matched samples from each pseudo-molecule of $F$. vesca, were selected from the 144 samples, and subjected to subsequent sequencing analysis to obtain approximately 4.3 million sequence reads per each sample on average (Additional file 1: Table S1). The reads were mapped on the reference sequences of the genomes of $F \times$ ananassa (FAN_r1.1), F. vesca (vasca_v2.0a1), and F. iinumae (FII_r1.1), respectively. Average alignment rates were different among the three references with $58 \%$ in $F . \times$ ananassa, $50 \%$ in F. vesca, and $34 \%$ in F. iinumae (Additional file 1: Table S1). Expectedly, in accordance with the alignment rate in each sample, it might be possible to distinguish the origins of the chromosome samples into two types (Fig. 5), Fv (alignment rates on $F$. vesca were similar to that on $F . \times$ ananassa) and non-Fv (alignment rates on $F$. vesca were similar to that on $F$. iinumae). The results suggested that homoeologous chromosomes were successfully isolated with our microdissection technique.
In order to enable a more complete understanding of the results of the mapping with pseudo-molecules in $F$. vesca, some example results are shown in Fig. 6. The reads of FaMD-1-C8 best matched with approximately 42\% of the sequence of Fvb1. Further, FaMD-3-E7, FaMD-2-D3, FaMD-2-C12, FaMD-4-C9, FaMD-6-E5, and FaMD-2-C1 were best matched at approximately $45 \%$ for Fvb2, 50\% for Fvb3, 40\% for Fvb4, 30\% for Fvb5, 60\% for Fvb6, and $30 \%$ for Fvb7, respectively. In each sample, approximately 1.5-4 million reads that matched the specific pseudomolecule in $F$. vesca were obtained. In contrast, approximately $10-20 \%$ of the reads in each sample matched with other pseudo-molecules. Possible explanations for the observed results are as follows. First, the pseudo-molecules might have some incorrectly assigned segments in the sequence because $F$. vesca [27] and $F$. $\times$ ananassa [9, $14]$ have $20.74-47.1 \%$ repeat sequences in their genomes. Although the assignment of repeat sequences might be problematic, our results indicate that a large percentage of the DNA reads derived from each single chromosome of cultivated strawberry could correspond to a specific pseudo-molecule of $F$. vesca. Second, each chromosome of the cultivated strawberry may contain small segments of the chromosomal DNA derived from each pseudomolecule of $F$. vesca, based on the evolutionary history of the cultivated strawberry. Third, DNA segments of the other chromosomes might have been contaminated in the PCR tube when the chromosome microdissection 


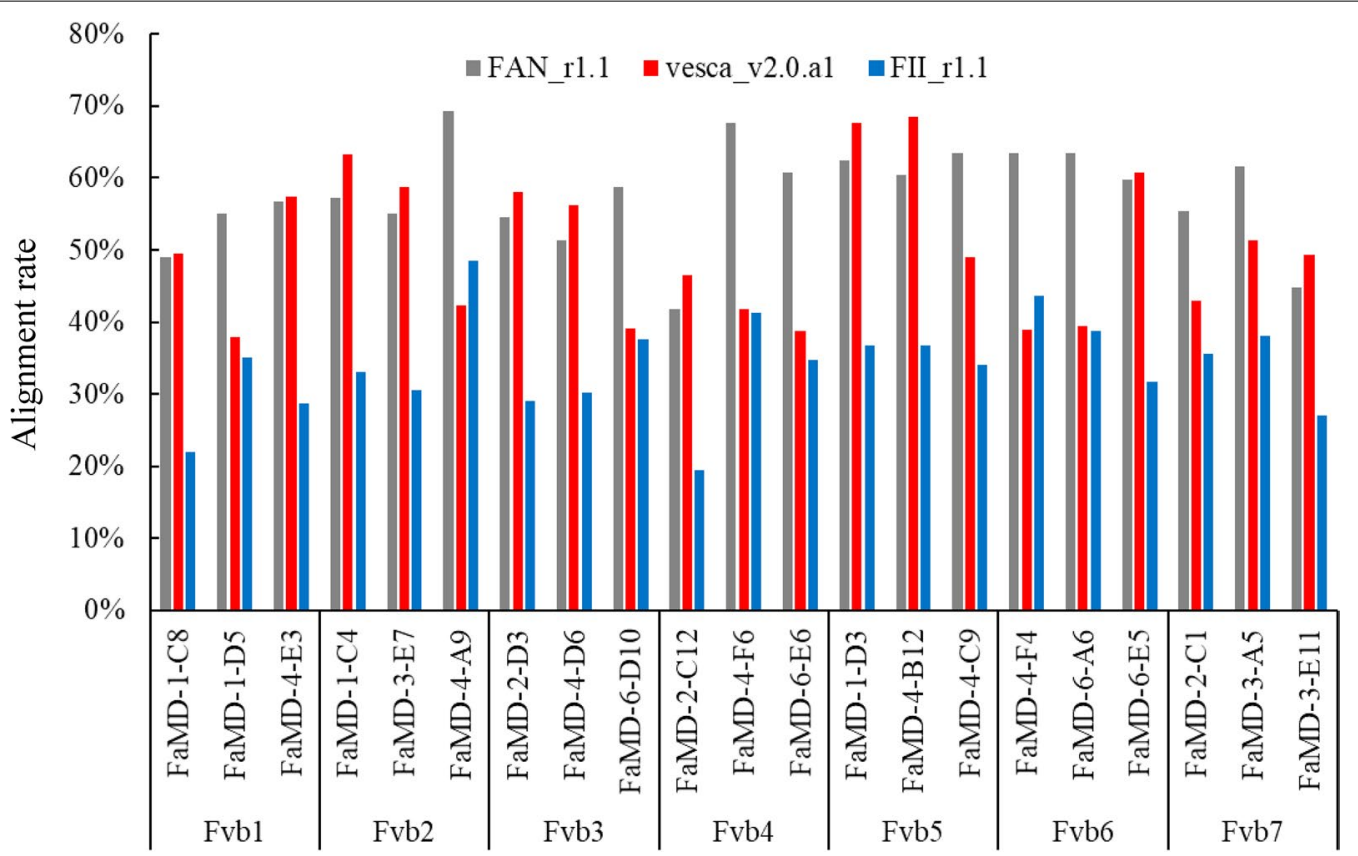

Fig. 5 Alignment rates of sequence reads obtained from single-chromosome samples onto the reference sequences of the genomes of F. $\times$ ananassa (FAN_r1.1), F. vesca (vasca_V2.0.a1), and F. iinumae (FII_r.1.1)

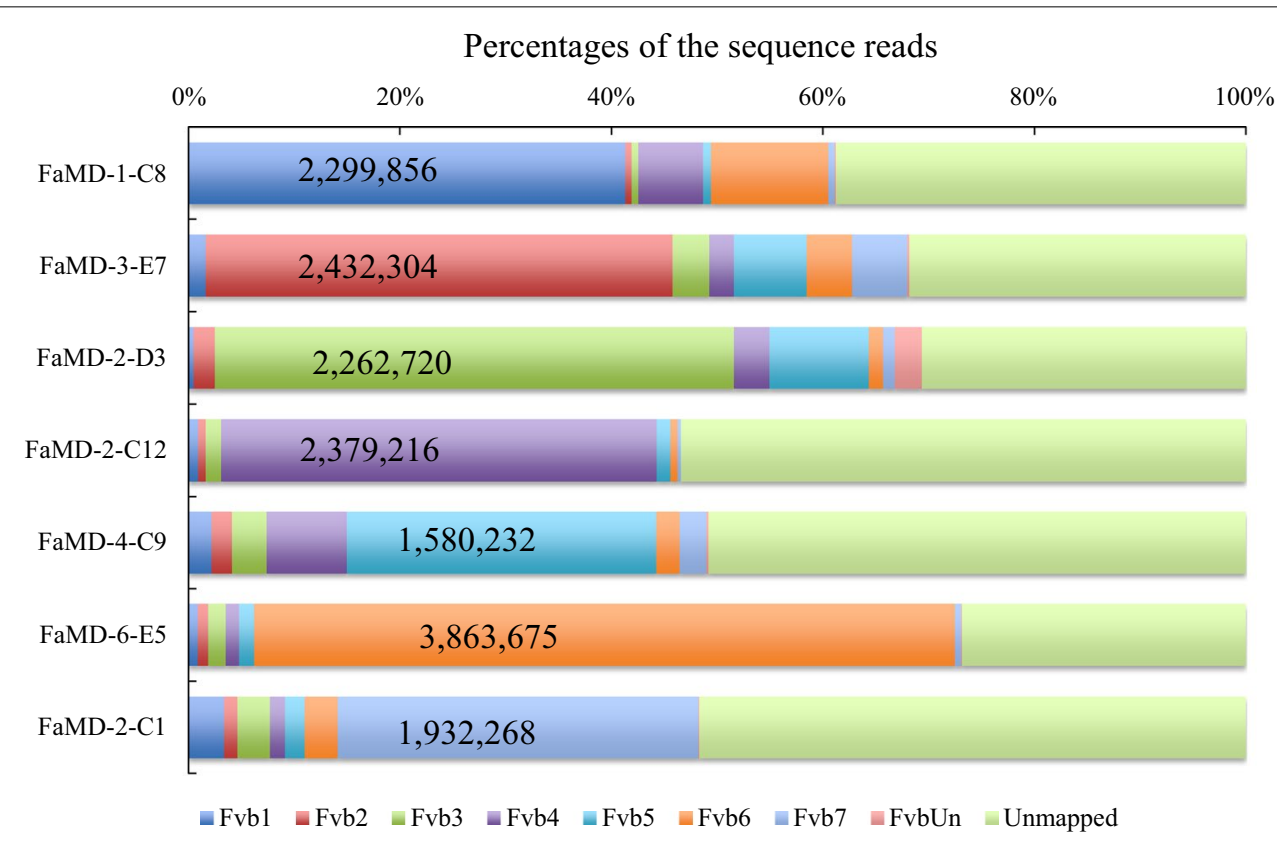

Fig. 6 Results of best-matched examples obtained by mapping onto pseudo-molecules of F. vesca $(2 n=14)$, that is one of the genome donors in the cultivated strawberry. The DNA segments that were obtained from a micro-dissected single chromosome, decoded the sequences by the NGS. Every DNA sequence was classified into Fvb1 to Fvb7 of pseudo-molecules in F. vesca, FvUn of unassigned sequence in F. vesca and unmapped one. In each bar, the number of the reads were exhibited 
was conducted. However, the possibility of such contamination is low, because we precisely microdissected a single chromosome. In addition, the total percentages of the matched reads to the other pseudo-molecules exceeded the level of potential contamination.

\section{Coverage rate of the amplified DNA from single chromosomes of cultivated strawberry}

To determine the percentage of the pseudo-molecules that were covered by the DNA sequence amplified from a single chromosome of cultivated strawberry, the coverage rate was determined for three sample data sets in each pseudo-molecule of $F$. vesca (Fig. 7). The coverage rates varied from 3 to $29.9 \%$. Although the coverage rates of the three samples were greater than $20 \%$, those of the remaining 18 samples were less than $12 \%$. This finding could be explained by the possibility that some chromosomes might have been partially microdissected or that the amplification might have been biased for some reason. In addition, the wide variation may be related to genome composition. As indicted in some papers [7-10], these models specified the presence of just two copies of the $F$. vesca-affiliated A (or Av) subgenome. Thus, if a chromosome in the A (or Av) genome was microdissected and amplified the coverage rates might increase but decrease in the other subgenomes. Currently, it is difficult to clearly determine the cause; however, the coverage rate could be as high as $30 \%$ for a single chromosome. On the basis of these results, if the $30 \%$ coverage rate were to be applied to each chromosome sample, it would be unnecessary to perform microdissection of many chromosomes to elucidate the entire DNA sequence of each chromosome. For this reason, the experimental method must be improved to increase the coverage rate. However, the entire DNA sequence that corresponds to chromosomal DNA in cultivated strawberry may be revealed through chromosome microdissection using the micromanipulation system.

\section{Conclusions}

The present study was conducted to determine the effectiveness of sequence analysis using single chromosomes for a typical allopolyploid species, cultivated strawberry. Then, the new efficient method to amplify DNA segments from a microdissected single somatic chromosome were exhibited. In addition, it confirmed that the amplified DNA segments were derived from the chromosome of strawberry plants by sequence analysis. The coverage rates of the DNA sequences from the single chromosome onto all pseudo-molecular sequences of the diploid $F$. vesca genome varied from 3 to $29.9 \%$. On the basis of these results, we believe that whole-genome analysis of allopolyploid plants can be enhanced using methodology that employs microdissected single chromosomes.

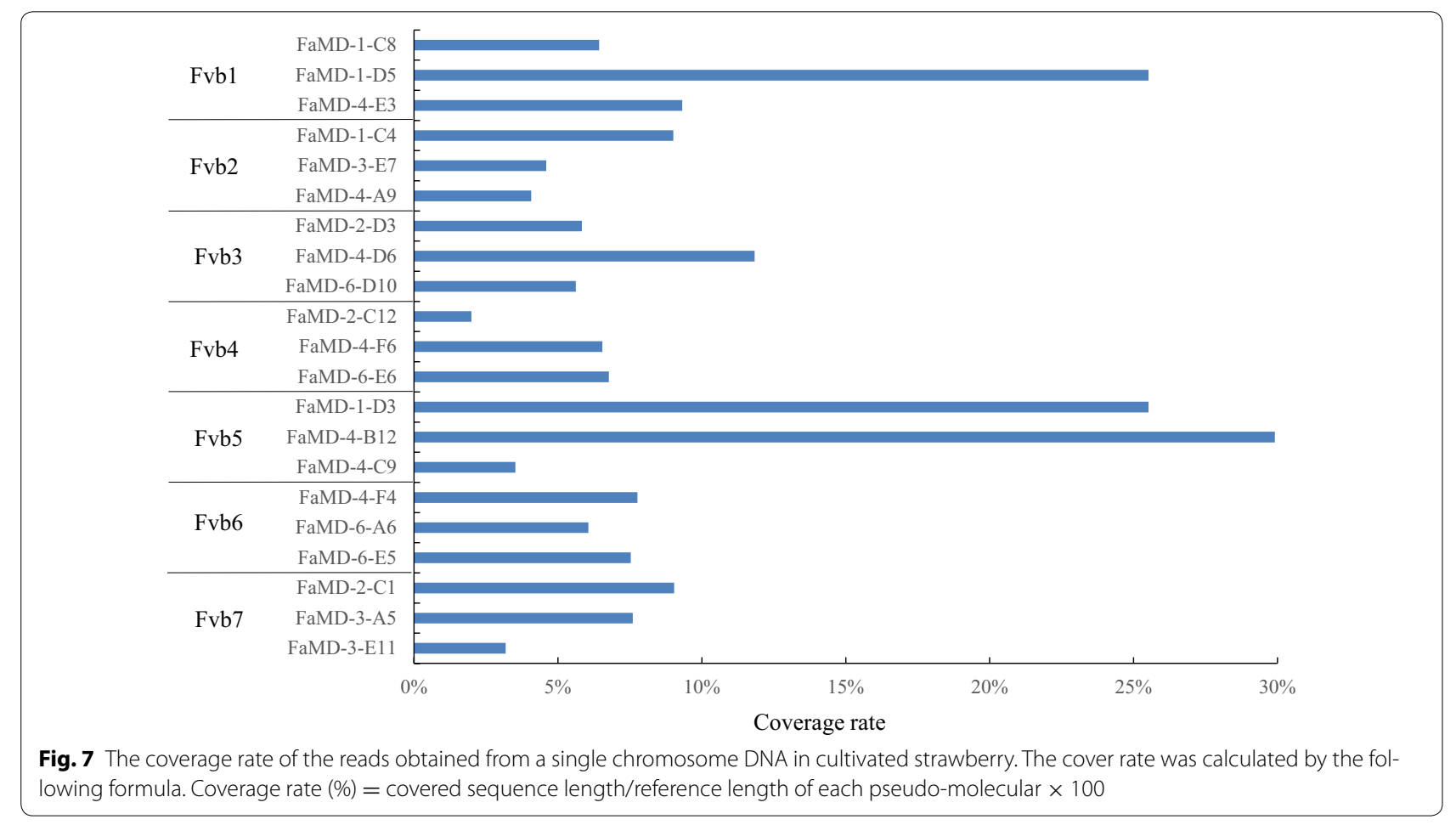




\section{Methods}

\section{Chromosome slide sample preparation}

Some newly propagated plants of a Japanese octoploid strawberry 'Reiko' that was grown in a greenhouse condition were used for the experiment. Pretreatment and fixation of root tips were conducted using a modified version of the method described by Iwatsubo and Naruhashi $[28,29]$, Nathewet et al. [4, 5], and Yanagi and Noguchi [30]. Root tips were collected at 17:00, pretreated with $2 \mathrm{mM}$ 8-hydroxyquinoline solution for $1 \mathrm{~h}$ at approximately $20{ }^{\circ} \mathrm{C}$, and subsequently maintained in the same solution at $4{ }^{\circ} \mathrm{C}$ for $15 \mathrm{~h}$ until 09:00 the following morning. The root tips were then fixed in a 1:3 (v:v) solution of acetic acid and ethanol for $40 \mathrm{~min}$ at room temperature. The fixed roots were trimmed to $2-3 \mathrm{~mm}$ from the tip and were softened using an enzyme cocktail, containing 4\% cellulase Onozuka RS (Yakult Co. Ltd., Tokyo), 0.3\% pectolyase Y-23 (Seishin Pharmaceutical Co. Ltd., Tokyo), 2.1\% macerozyme R10 (Yakult Co. Ltd., Tokyo), and $1 \mathrm{mM}$ EDTA pH 4.2 at $37{ }^{\circ} \mathrm{C}$ for $25 \mathrm{~min}$. Subsequently, the roots were rinsed twice in distilled water. Then one root tip that was selected using a Pasteur pipette with distilled water and placed in the center of a glass slide. After eliminating the water, $10 \mu \mathrm{L} 45 \%$ acetic acid was placed on the root tip, followed by incubation for $2 \mathrm{~min}$ and then maceration using forceps. A cover slip was placed on the preparation, tapped gently with a chopstick, heated using an alcohol lamp for a few seconds, and then pressed with a thumb. The glass slide was exposed to $-80^{\circ} \mathrm{C}$ for at least $5 \mathrm{~min}$ in an ultra-low temperature freezer, and then the cover slip was removed using a razor blade at room temperature. The slide samples were dipped in a $70 \%$ alcohol solution at $-20{ }^{\circ} \mathrm{C}$ prior to microdissection.

\section{Chromosome microdissection}

Chromosome microdissection was conducted under a light microscope (BX51; Olympus Co.), which was equipped with a micromanipulation system (MN-4 and MMO-203; Narishige Co.) and a long focus objective lens ( $\times 50$ SLMPLN; Olympus Co.). A glass needle for picking up a single chromosome of the cultivated strawberry was fabricated using a glass puller device (PC-10; Narishige Co.). Chromosome microdissection was conducted in a clean room to avoid DNA contamination by atmospheric microorganisms. Single chromosomes on the sample slide were selected individually. After confirming the presence of a single chromosome at the tip of the glass needle under the light microscope, it was placed in a PCR tube containing $1 \mu \mathrm{L} 1 \times$ PBS buffer. The tip of the glass needle to which the microdissected single chromosome was adhered was then pressed against the bottom of the PCR tube and folded, and both were placed in the PCR tube. In total, 310 sample PCR tubes with single chromosomes were prepared from 10 somatic cells of cultivated strawberry.

\section{DNA amplification and analysis by NGS}

DNA amplification was conducted using an Illustra Single Cell GenomiPhi DNA Amplification kit (GE Healthcare Co.), according to the manufacturer's protocol. After adding $1 \mu \mathrm{L}$ lysis buffer, the PCR tube was heated at $65^{\circ} \mathrm{C}$ for $10 \mathrm{~min}$. To the PCR tube, $11 \mu \mathrm{L}$ reaction buffer, $1 \mu \mathrm{L}$ enzyme mix, $1 \mu \mathrm{L}$ amplification mix, and $4 \mu \mathrm{L}$ sterile water were then added. The PCR tube was incubated at $30{ }^{\circ} \mathrm{C}$ for $180 \mathrm{~min}$, and subsequently heated at $65{ }^{\circ} \mathrm{C}$ for $10 \mathrm{~min}$ to inactivate the enzymes. Following amplification, the concentration of the DNA was measured using a fluorometer (Qubit ${ }^{\circledR}$ 3.0; Thermo Fisher Co.). The DNA was then fragmented using a DNA Shearing Tube g-TUBE (Covaris, Woburn, MA, USA) or NEBNext dsDNA Fragmentase (New England Biolabs, Hitchin, UK) into lengths of approximately $600 \mathrm{bp}$ for sequencing library preparation using a TruSeq Nano DNA Sample Prep Kit (Illumina, San Diego, CA, USA). The nucleotide sequences of the libraries were determined using a MiSeq system (Illumina) in paired-end mode (301-base) or a NextSeq 500 system (Illumina) in paired-end mode (151-base). The sequence reads were submitted in the DDBJ Sequence Read Archive under the accession number DRA005991.

\section{Data processing and mapping}

Low-quality sequences were removed and adapters were trimmed using PRINSEQ [31] and fastx_clipper in the FASTX-Toolkit (http://hannonlab.cshl.edu/fastx toolkit). Sequence similarity searches of 1000 randomly selected reads from each library were performed against the NCBI nt (non-redundant nucleotide sequences) database (http://www.ncbi.nlm.nih.gov), the $F$. vesca genome, v2.0.a1 [9], the cultivated strawberry genome, FAN_r1.1 [14], the $F$. vesca chloroplast genome (Accession number NC_015206), and the A. thaliana mitochondrion genome (Accession number NC_001284) using the BLASTN program with an $E$ value cutoff of $\leq 1 e^{-10}$ [32]. Furthermore, all of the filtered reads were mapped onto the $F$. vesca genome (version v2.0.a1) and the cultivated strawberry genome (FAN_r1.1) as reference sequences using Bowtie 2 [33]. The resulting sequence alignment/map format files were converted to binary sequence alignment/map format (BAM) files. Genome coverage was calculated from the BAM files using the BEDtools script genomeCoverageBed [34].

\section{Additional file}

Additional file 1: Table S1. Numbers and alignment rates of sequence reads obtained from single-chromosome samples. 


\section{Authors' contributions}

TY, KS, MT, and SI designed the study, performed experiments, and analyzed the data. MT microdissected the chromosomes. TY, KS, and SI wrote the manuscript. All authors read and approved the final manuscript.

\section{Author details}

${ }^{1}$ Faculty of Agriculture, Kagawa University, Miki-cho, Kita-gun, Kagawa 761-0795, Japan. ${ }^{2}$ Kazusa DNA Research Institute, Kazusa-Kamatari, Kisarazu, Chiba, Japan.

\section{Acknowledgements}

We are grateful to Ms. F. Maeda (Chiba Prefectural Agriculture and Forestry Research Center, Japan) for providing the plant material, to Dr. H. Masumoto (Kazusa DNA Research Institute) for his kind support, and to Dr. T. Kornlawat (Kagawa University) and S. Sasamoto, C. Minami, and S. Nakayama (Kazusa DNA Research Institute) for their technical assistance.

\section{Competing interests}

The authors declare that they have no competing interests.

\section{Availability of data and materials}

The datasets supporting the conclusions of this article are included within the article.

\section{Consent for publication}

All authors have given consent for the manuscript.

\section{Ethics approva}

Not applicable.

\section{Funding}

This work was supported by the Science and Technology Research Promotion Program for Agriculture, Forestry, Fisheries and Food industry in Ministry of Agriculture, Forestry and Fisheries in Japan (27003A).

\section{Publisher's Note}

Springer Nature remains neutral with regard to jurisdictional claims in published maps and institutional affiliations.

Received: 18 May 2017 Accepted: 8 October 2017

Published online: 30 October 2017

\section{References}

1. FAOSTAT. http://www.fao.org/faostat/en/\#home. Accessed 11 May 2017.

2. Ichijima K. Cytological and genetic studies on Fragaria. Genetics. 1926;11:590-604.

3. Longley AE. Chromosomes and their significance in strawberry classification. J Agric Res. 1926;15:559-68.

4. Nathewet P, Yanagi T, Sone K, Taketa S, Okuda N. Chromosome observation method at metaphase and pro-metaphase stages in diploid and octoploid strawberries. Sci Hortic. 2007;114:133-7.

5. Nathewet P, Yanagi T, Iwastubo Y, Sone K, Takamura T, Okuda N. Improvement of staining method for observation of mitotic chromosome in octoploid strawberry plants. Sci Hortic. 2009;120:431-5.

6. Fedorova NJ. Crossability and phylogenetic relations in the main European species of Fragaria. C R Acad Sci USSR. 1946;53:545-7.

7. Senanayake YDA, Bringhurst RS. Origin of the Fragaria polyploids. I. Cytological evidence. Am J Bot. 1967;54:221-8.

8. Bringhurst RS. Cytogenetics and evolution in American Fragaria. HortScience. 1990;25:879-81.

9. Tennessen JA, Govindarajulu R, Ashman T-L, Liston A. Evolutionary origins and dynamics of octoploid strawberry subgenomes revealed by dense targeted capture linkage maps. Genome Biol Evol. 2014;6:3295-313.

10. Sargent DJ, Yang Y, Surbanovski N, Bianco L, Buti M, Velasco R, Giongo L, Davis TM. Haplo SNP affinities and linkage map positions illuminate subgenome composition in the octoploid, cultivated strawberry (Fragaria $\times$ ananassa). Plant Sci. 2016;242:140-50. doi:10.1016/j. plantsci.2015.07.004.
11. Akiyama Y, Yamamoto Y, Ohmido N, Oshima M, Fukui K. Estimation of the nuclear DNA content of strawberries (Fragaria spp.) compared with Arabidopsis thaliana by using dual system flow cytometry. Cytologia. 2001;66:431-6.

12. Nathewet $P$, Hummer KE, Yanagi T, Iwatsubo Y, Sone K. Karyotype analysis in octoploid and decaploid wild strawberries in Fragaria (Rosaceae). Cytologia. 2010;75:277-88.

13. Van DijkT, Noordijk Y, Dubos T, Bink MCAM, Meulenbroek EJ, Visser RGF, Van de Weg WE. Microsatellite allele dose and configuration establishment (MADCE): an integrated approach for genetic studies in allopolyploids. BMC Plant Biol. 2012;12:25

14. Hirakawa H, Shirasawa K, Kosugi S, Tashiro K, Nakayama S, Yamada M, Kohara M, Watanabe A, Kishida Y, Fujishiro T, Tsuruoka H, Minami C, Sasamoto S, Kato M, Nanri K, Komaki A, Yanagi T, Qin G, Maeda F, Ishikawa M, Kuhara S, Sato S, Tabata S, Isobe SN. Dissection of the octoploid strawberry genome by deep sequencing of the genomes of Fragaria species. DNA Res. 2014;21:169-81.

15. Hagag N, Viola M. Microdissection and cloning: a practical guide. New York: Academic Press; 1993

16. Scalenghe F, Turco E, Ederström JE, Pirrotta V, Melli M. Microdissection and cloning of DNA from a specific region of Drosophila melanogaster polytene chromosomes. Chromosoma. 1981;82:205-16.

17. Zhou RN, Hu ZM. The development of chromosome microdissection and microcloning technique and its applications in genomic research. Curr Genomics. 2007;8:67-72.

18. Sandery MJ, Forster JW, Macadam SR, Blunden R, Jones RN, Brown SDM. Isolation of a sequence common to $\mathrm{A}$ and $\mathrm{B}$-chromosomes of rye (Secale cereale) by microcloning. Plant Mol Biol Rep. 1991;9:21-30.

19. Zhu T, Shi L, Keim P. Detection by in situ fluorescence of short, singlecopy sequences of chromosomal DNA. Plant Mol Biol Rep. 1995;13:270-7.

20. Capal P, Blavet N, Vrana J, Kubalakova M, Dolezel J. Multiple displacement amplification of the DNA from single flow-sorted plant chromosome. Plant J. 2015:84:838-44.

21. Saunders RD, Glover DM, Ashburner M, Siden-Kiamos I, Louis C, Monastirioti M, Savakis C, Kafatos F. PCR amplification of DNA microdissected from a single polytene chromosome band: a comparison with conventional microcloning. Nucleic Acid Res. 1989;17:9027-37.

22. Saunders RDC. Short cuts for genomic walking: chromosome microdissection and the polymerase chain reaction. BioEssays. 1990;12:245-8.

23. Lebo RV, Carrano AV, Burkhart-Schultz KJ, Dozy AM, Yu L-C, Kan YW. Assignment of human beta, gamma, and delta globin genes to the short arm of chromosome 11 by chromosome sorting and DNA restriction enzyme analysis. Proc Natl Acad Sci USA. 1979;76:5804-8.

24. Telenius H, Carter NP, Bebb CE, Nordenskjöld M, Ponder BAJ, Tunnacliffe A. Degenerated oligonucleotide-primed PCR: general amplification of target DNA by a single degenerate primer. Genomics. 1992;13:718-25.

25. Zhang YX, Deng CL, Hu ZM. The chromosome microdissection and microcloning technique. In: Kianian FS, Kianian PMA, editors. Plant cytogenetics: methods and protocols, methods in molecular biology, vol. 1429. New York: Springer; 2016. p. 151-60. doi:10.1007/978-1-4939-3622-9 12.

26. Seifertova E, Zimmerman LB, Gilchrist MJ, Macha J, Kubickova S, Cernohorska H, Zarsky V, Owens ND, Sesay AK, Tlapakova T, Krylov V. Efficient high-throughput sequencing of a laser microdissected chromosome arm. BMC Genomics. 2013;14:357.

27. Shulaev V, Sargent DJ, Crowhurst RN, Mockler TC, Folkerts O, Delcher AL, Jaiswal P, Mockaitis K, Liston A, Mane SP, Burns P, Davis TM, Slovin JP, Bassil N, Hellens RP, Evans C, Harkins T, Kodira C, Desany B, Crasta OR, Jensen RV, Allan AC, Michael TP, Setubal JC, Celton J-M, Rees JDG, Williams KP, Holt SH, Rojas JJR, Chatterjee M, Liu B, Silva H, Meisel L, Adato A, Filichkin SA, Troggio M, Viola R, Ashman T-L, Wang H, Dharmawardhana P, Elser J, Raja R, Priest HD, Bryant DW, Fox SE, Givan SA, Wilhelm LJ, Naithani S, Christoffels A, Salama DY, Carter J, Girona EL, Zdepski A, Wang W, Kerstetter RA, Schwab W, Korban SS, Davik J, Monfort A, Denoyes-Rothan B, Arus P, Mittler R, Flinn B, Aharoni A, Bennetzen JL, Salzberg SL, Dickerman AW, Velasco R, Borodovsky M, Veilleux RE, Folta KM. The genome of wood land strawberry (Fragaria vesca). Nat Genet. 2010;43:109-16.

28. Iwatsubo Y, Naruhashi N. Karyotypes of three species of Fragaria (Rosaceae). Cytologia. 1989;54:493-7.

29. Iwatsubo Y, Naruhashi N. Karyotypes of Fragaria nublicola and F. daltoniana (Rosaceae). Cytologia. 1991;56:453-7. 
30. Yanagi T, Noguchi Y. Strawberry (Plants in the genus Fragaria). In: Mason AS, editor. Polyploidy and hybridization for crop improvement. Florida: CRC Press; 2016. p. 115-58.

31. Schmieder R, Edwards R. Quality control and preprocessing of metagenomic datasets. Bioinformatics. 2011;27:863-4.

32. Altschul SF, Madden TL, Schaffer AA, Zhang J, Zhang Z, Miller W, Lipman DJ. Gapped BLAST and PSI-BLAST: a new generation of protein database search programs. Nucleic Acid Res. 1977;25:3389-402.
33. Langmead B, Salzberg SL. Fast gapped-read alignment with Bowtie 2. Nat Methods. 2012;9:357-9.

34. Quinlan AR, Hall IM. BEDTools: a flexible suite of utilities for comparing genomic features. Bioinformatics. 2010;26:841-2.
Submit your next manuscript to BioMed Central and we will help you at every step:

- We accept pre-submission inquiries

- Our selector tool helps you to find the most relevant journal

- We provide round the clock customer support

- Convenient online submission

- Thorough peer review

- Inclusion in PubMed and all major indexing services

- Maximum visibility for your research

Submit your manuscript at www.biomedcentral.com/submit
() Biomed Central 